\title{
Factors that Influence Quiet Standing Balance of Patients with Incomplete Cervical Spinal Cord Injuries
}

\author{
Ga Eun Lee, M.D., Hasulk Bae, M.D. ' , Tae Silk Yoon, M.D. ', Joo Sup Kim, M.D. ${ }^{2}$, \\ Tae Im Yi, M.D. ${ }^{2}$, Jun Sung Park, M.D. ${ }^{2}$
}

Department of Rehabilitation Medicine, Daegu Workers' Compensation Hospital, Daegu 702-911,

${ }^{1}$ Ewha Womans University College of Medicine, Seoul 158-710, ${ }^{2}$ Bundang Jesaeng General Hospital, Seongnam 463-774, Korea

Objective To investigate the factors influencing the quiet standing balance of patients with incomplete cervical spinal cord injuries. Also to find the correlations between posturographic parameters and clinical balance tests as well as to find the correlation between posturographic parameters and functional independence.

Method We conducted a tetra-ataxiometric posturography, lower extremity motor score (LEMS), Korean version of the Berg Balance Scale (K-BBS), Timed Up and Go test (TUG), and Korean Version of the Modified Barthel Index (K-MBI) of 10 patients. 10 healthy adults carried out the posturography. We checked stability, weight distribution, Fourier and synchronization indices of eight positions, and the fall index of the posturography.

Results The patient group showed significantly higher stability and weight distribution indices in all eight positions. Stability indices significantly increased with eyes closed or standing on pillows. Weight distribution indices were significantly higher with eyes closed or the head bent backwards. The patient group showed significantly higher Fourier indices of low, low-medium, and high frequency in eight positions. The Fourier indices at high-medium frequency were significantly higher with eyes closed on pillows or in variable head positions. There were no significant differences of synchronization indices between the patient and the control group. The falling index of the patient group significantly correlated with K-BBS, TUG, and K-MBI. LEMS had significant correlation with some synchronization indices, but not with the falling index.

Conclusion The quiet standing balance of the patients was influenced by somatosensory limitations or insufficient visual compensation. We should try to improve the postural balance and functional independence of patients through proper proprioceptive and lower extremity strength training for better postural and pedal control, and to make efforts to minimize environmental hazards.

Key Words Spinal cord injury, Balance, Posture, Barthel index

Received November 14, 2011; Accepted May 18, 2012

Corresponding author: Ga Eun Lee

Department of Rehabilitation Medicine, Daegu Workers' Compensation Hospital, 515, Hakjeong-ro, Buk-gu, Daegu 702-911, Korea

Tel: +82-53-715-7575, Fax: +82-53-715-7722, E-mail: smart0416@hotmail. com

(c) This is an open-access article distributed under the terms of the Creative Commons Attribution Non-Commercial License (http:// creativecommons.org/licenses/by-nc/3.0) which permits unrestricted noncommercial use, distribution, and reproduction in any medium, provided the original work is properly cited.

Copyright $\odot 2012$ by Korean Academy of Rehabilitation Medicine

\section{INTRODUCTION}

One of the primary goals for patients with incomplete cervical spinal cord injuries is to regain walking ability. ${ }^{1}$ Locomotor training, as a rehabilitation strategy, focuses on postural control, balance, standing, walking, health, and the quality of life after neurologic injury. ${ }^{2}$ It is frequently seen that patients with the same level of injury, or the same American Spinal Cord Injury Association 
(ASIA) impairment scale score, have different stabilities or walking abilities. We considered that this is because of the possible differences in balance control, but there is little data. ${ }^{3}$

Balance is very important to maintain a sitting position and to move from a sitting to a standing position (sit to stand). Improving a standing balance and weight-bearing regulation is vital to improve walking ability. ${ }^{4}$ Balance is maintained by the integration and regulation of information from the somatosensory, visual, and vestibular systems, and by the reflexive control of limbs. ${ }^{5,6}$ The spinal cord is a pathway for the somatosensory organs. Spinal cord injuries result in maintaining balance difficulty. The balance of people with spinal cord injuries seems to be affected by the impairment of proprioception after an injury to the central nervous system. However, current research to define which element of the balance system are affected, and how much extent of proprioception are impaired is limited.

Even though balance control can be assessed clinically using the Berg Balance Scale (BBS), the functional reach test, and the Timed Up and Go test (TUG), it is difficult to assess the diversity of postural control. ${ }^{7}$ Recently posturography has been used to help assess this diversity. The tetra-ataxiometric posturography has 4 separate force-plates, one for each forefoot and heel. It can quantify the postural control of patients through various positions with visual and somatosensory changes. Diverse factors like stability indices and weight distribution patterns are calculated by analyzing the trajectories of the pressures from each of the force-plates. ${ }^{8,9}$

Using posturography, this study assessed the factors that influence the quiet standing balance of patients with incomplete cervical spinal cord injuries and compared them with a healthy control group. We attempted to discover whether the balance index of the patients had any significant correlation with the strength of their lower extremities, using the K-BBS, and TUG. We also analyzed the correlation between balance and activities of daily living.

\section{MATERIALS AND METHODS}

We included 10 patients with incomplete spinal cord injuries under 60 years old, who could stand alone and walk over $5 \mathrm{~m}$, with or without assistive devices, and per- form simple commands. The control group contained 10 healthy people without cervical spinal cord injury. We excluded people with severe medical illnesses, cystitis and other infections, thromboembolic diseases, severe contracture or spasticity of lower extremities, visual or peripheral vestibular impairments, and individuals taking medicine that affected their balance.

The patient group, comprised of 8 men and 2 women, possessed the following characteristics: mean age $46.7 \pm$ 8.5 years, height $169.7 \pm 8.0 \mathrm{~cm}$, weight $67.0 \pm 7.0 \mathrm{~kg}$, and onset duration $2.7 \pm 6.3$ years. They had the impairment of pain, temperature and proprioception below the neurologic level of injury with perianal and deep anal senses preserved. The onset latency of the $\mathrm{P} 1$ potential in the sensory evoked potential (EP) test was more significantly delayed than the control group. The control group included 5 men and 5 women whose mean age was $42.2 \pm 10.2$ years, the height was $166.1 \pm 7.3 \mathrm{~cm}$, and weight was $62.6 \pm 9.1 \mathrm{~kg}$. There were no significant differences in age, gender, height, and weight compared with those of the patient group.

Lower-extremity strength of the patient group was evaluated using the lower extremity motor score (LEMS). The power of 5 key muscles was rated using a 6 point scale, rating from 0-5. "0" indicated no contraction and " 5 " a normally strong contraction. The total possible score of LEMS was 50 points.

The clinical balance tests of the patients included the Korean version of the Berg balance scale (K-BBS), and the Timed Up and Go test (TUG). K-BBS is a 14-item scale designed to measure standing balance and sit-to-stand positions. Scores are based on a 5 point scale, rating from $0-4$. " 0 " indicates the lowest level of function and " 4 " the highest level of function. The total possible score is 56 points. Each item consists of actions related to activities of daily living. ${ }^{10}$ It has been used as a tool to evaluate the balance of patients with spinal cord injuries, and has significant correlations not only with ambulatory scales like the walking index for spinal cord injury (WISCI), ${ }^{3,11}$ and 50 -foot walking speed 11 but also the locomotor scale functional independent measure (LFIM), and total FIM. ${ }^{11}$

The TUG measures, in seconds, the time a person requires to stand up from a standard armchair, walk $3 \mathrm{~m}$, turn, walk back to the chair, and sit down again. ${ }^{2,12} \mathrm{~A}$ practice trial is given, followed by 2 timed trials. The results of the timed trials are averaged. It estimates the in- 
creased risk of fall, functional mobility, and the ability to transfer. TUG has a high reliability and significant correlation with BBS. ${ }^{7}$ It is significantly correlated with WISCI II in people with spinal cord injuries. ${ }^{13}$ Faster TUG times indicated improved gait speed and endurance. ${ }^{14}$

The 10-item Korean version of the Modified Barthel Index (K-MBI) measured the functional ability to perform activities of daily living, and the degree of independence of the patients. The 10-items were feeding, grooming, bathing, dressing, bladder and bowel care, toilet use, ambulation, transfer and stairs. The maximal score was 100 , indicating the patient was fully independent in physical functioning. The lowest score was 0 , representing a totally dependent bedridden state. ${ }^{15,16}$

We used Tetrax ${ }^{\circledR}$ (Sunlight Medical Ltd., Ramat Gan, Israel) to check various factors that influence the quiet standing balance of the patient and control groups (Fig. 1). Tetrax ${ }^{\circledR}$ software analyzed pressure information transmitted from the four foot-plates. A practice trial was given, followed by 1 timed trial for postural stabilization. Each trial was performed at 8 different positions. Candidates placed their feet on the foot-prints of the footplates, and restricted unnecessary movement. The 8 positions included: standing with eyes open/closed, on foam-rubber pillows with eyes open/closed, with eyes closed and head turned right/left/backward/forward.

Tetrax ${ }^{\circledR}$ parameters are: stability index, weight distribution index, Fourier index, synchronization index, and fall index.
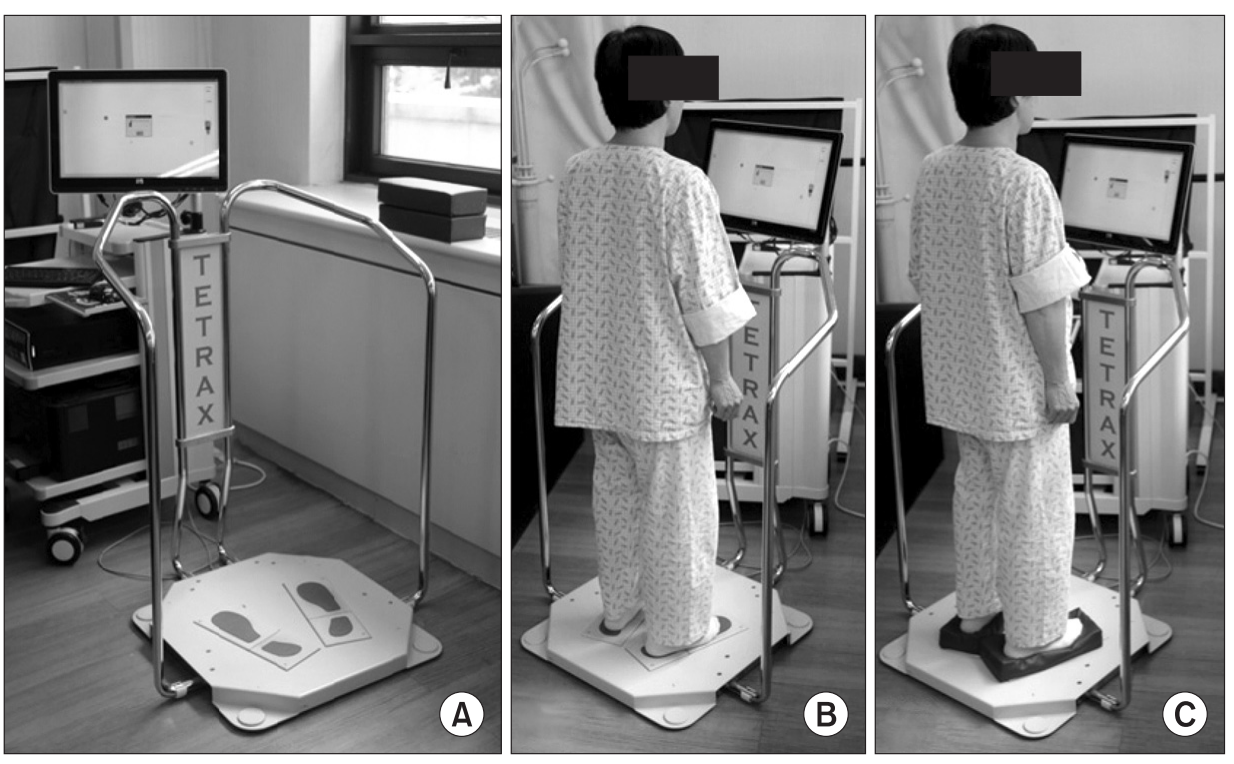

Fig. 1. Tetrax $^{\circledR}$ (Sunlight Medical Ltd., Ramat Gan, Israel) is composed of monitor, desktop, four force platforms, and foam-rubber pillows (A). It was used to evaluate the postural balance (B). The patient was standing on pillows (C).
The stability index measured the agitation on the four plates in order to measure the overall stability and assess how well the subject could control and compensate for postural changes. The higher the stability index was, the more unstable the subject was.

The weight distribution index indicated the weight distribution on the foot-plates, and its normal range was 4-6. The ideal posture was when $25 \%$ of the subjects' weight was placed on each plate. The index became larger, with increased deviation from the ideal. A very high index indicated the subject may have orthopedic or neurologic disorders, a very low index indicated possible postural rigidity. induced by regression analysis, which was a Fouriertransformation of the frequency of postural sway. A higher index suggested the possibility of a pathologic state. Subjects with a well-controlled balance showed a highintensity, at $0.1 \mathrm{~Hz}$ (low frequency). This might mean that postural stability was controlled by normal transmission tural sway at a low frequency related to minimal effort and less stress to maintain a quiet standing balance as long as possible. Postural sway at $0.1-0.5 \mathrm{~Hz}$ (low-medium frequency) might have something to do with vestibular impairment, musculoskeletal fatigue, or alcohol ingestion. Postural sway at $0.5-1 \mathrm{~Hz}$ (high-medium frequency) might relate to somatosensory impairment of lowerextremities. Postural sway at over $1 \mathrm{~Hz}$ (high frequency)
The Fourier index showed the intensity of postural sway and feedback systems of visual-vestibular signals. Pos- 
might be due to lesions of the central nervous system.

The synchronization index compared the vibration waves of two out of the four plates. The index between the toe and heel plates had a negative value, because there was a compensatory movement and mirror-image compensation between those foot-plates. The index between two toe-plates, or two heel-plates, had a positive value because the movement was simultaneous and the synchronization was parallel. The index ranged from $-1,000$ to 1,000 . If the absolute value of the index was lower than 200, it meant that the subject had a lack of fine pedal control.

The fall index was calculated by the sum of the standard deviations of stability, Fourier transformation, and synchronization indices. It has been shown to be related to the risk of falling. A higher index reflected an increased risk of falling. ${ }^{8,9,17}$

SPSS 17.0 was used for statistical analysis. The significance of characteristics between the two groups was tested with a Mann-Whitney test. The significances of the stability and weight distribution indices at different positions were tested with a one-way ANOVA. In addition, if there was a significant difference, multiple comparisons were performed. The correlations between the balance and LEMS, K-BBS, TUG, and K-MBI were tested using a Pearson correlation. Differences were considered statisti-

Table 1. Comparison of Stability Indices and Weight Distribution Indices at Eight Positions between the Patients and Control Group

\begin{tabular}{lccccc}
\hline \multirow{2}{*}{ Position } & \multicolumn{2}{c}{ Stability index } & & \multicolumn{2}{c}{ Weight distribution index } \\
\cline { 2 - 3 } \cline { 5 - 6 } & Patient & Control & & Patient & Control \\
\hline Eyes open & $22.2 \pm 8.0^{*}$ & $10.5 \pm 3.7$ & & $4.7 \pm 1.6^{*}$ & $2.6 \pm 0.9$ \\
Eyes closed & $34.6 \pm 19.0^{*}$ & $17.3 \pm 5.5$ & & $6.4 \pm 3.1^{*}$ & $2.5 \pm 1.2$ \\
Eyes open on pillows & $23.4 \pm 6.8^{*}$ & $14.8 \pm 4.0$ & & $4.5 \pm 2.4^{*}$ & $2.2 \pm 0.8$ \\
Eyes closed on pillows & $53.1 \pm 19.4^{*}$ & $27.7 \pm 5.1$ & & $6.9 \pm 3.4^{*}$ & $2.5 \pm 1.0$ \\
Head right & $37.8 \pm 21.2^{*}$ & $15.9 \pm 5.1$ & & $6.4 \pm 2.8^{*}$ & $2.5 \pm 0.9$ \\
Head left & $37.8 \pm 18.4^{*}$ & $16.3 \pm 3.3$ & & $6.1 \pm 2.3^{*}$ & $3.2 \pm 1.0$ \\
Head backward & $39.0 \pm 15.0^{*}$ & $19.5 \pm 5.5$ & & $8.4 \pm 3.3^{*}$ & $2.9 \pm 1.8$ \\
\hline Head forward & $39.7 \pm 24.1^{*}$ & $18.1 \pm 5.1$ & & $5.8 \pm 1.7^{*}$ & $2.7 \pm 1.3$ \\
\hline
\end{tabular}

Values are mean \pm standard deviation

Post-hoc Tukey test was applied among the different positions following a significance on the one-way ANOVA $\left({ }^{*} \mathrm{p}<\right.$ $0.05)$

Table 2. Comparison of Fourier Transformations in Eight Positions between the Patient and Control Group

\begin{tabular}{|c|c|c|c|c|c|c|c|c|}
\hline \multirow[t]{2}{*}{ Position } & \multicolumn{2}{|c|}{ Low frequency } & \multicolumn{2}{|c|}{$\begin{array}{l}\text { Low-medium } \\
\text { frequency }\end{array}$} & \multicolumn{2}{|c|}{$\begin{array}{l}\text { High-medium } \\
\text { frequency }\end{array}$} & \multicolumn{2}{|c|}{ High frequency } \\
\hline & Patient & Control & Patient & Control & Patient & Control & Patient & Control \\
\hline Eyes open & $31.0 \pm 20.6^{*}$ & $11.4 \pm 4.0$ & $10.1 \pm 7.5^{*}$ & $3.2 \pm 2.4$ & $2.1 \pm 1.9$ & $1.0 \pm 0.5$ & $1.0 \pm 0.5^{*}$ & $0.5 \pm 0.3$ \\
\hline Eyes closed & $23.2 \pm 10.1^{*}$ & $13.1 \pm 5.5$ & $11.8 \pm 1.0^{*}$ & $3.1 \pm 2.8$ & $2.7 \pm 2.1$ & $1.3 \pm 1.2$ & $1.4 \pm 0.9^{*}$ & $0.7 \pm 0.2$ \\
\hline Eyes open on pillows & $30.8 \pm 11.3^{*}$ & $15.7 \pm 6.8$ & $9.2 \pm 0.3^{*}$ & $2.4 \pm 4.2$ & $1.4 \pm 1.0$ & $1.1 \pm 0.7$ & $1.2 \pm 0.5^{*}$ & $0.7 \pm 0.2$ \\
\hline Eyes closed on pillows & $30.5 \pm 9.7^{*}$ & $18.6 \pm 11.3$ & $9.2 \pm 11.7$ & $3.9 \pm 3.5$ & $3.8 \pm 2.6^{*}$ & $1.8 \pm 1.4$ & $2.4 \pm 1.0^{*}$ & $1.4 \pm 0.3$ \\
\hline Head right & $27.7 \pm 12.3^{*}$ & $11.9 \pm 5.9$ & $10.7 \pm 4.2^{*}$ & $3.3 \pm 1.8$ & $2.5 \pm 2.4^{*}$ & $0.8 \pm 0.5$ & $1.7 \pm 1.1^{*}$ & $0.8 \pm 0.4$ \\
\hline Head left & $32.9 \pm 10.1^{*}$ & $13.8 \pm 7.4$ & $9.5 \pm 2.4^{*}$ & $1.7 \pm 2.5$ & $3.4 \pm 3.5^{*}$ & $1.0 \pm 0.8$ & $1.8 \pm 1.1^{*}$ & $0.8 \pm 0.2$ \\
\hline Head backward & $27.7 \pm 10.5^{*}$ & $15.5 \pm 10.2$ & $16.3 \pm 8.6^{*}$ & $2.5 \pm 3.4$ & $2.5 \pm 1.8^{*}$ & $1.1 \pm 0.5$ & $1.9 \pm 0.9 *$ & $0.9 \pm 0.2$ \\
\hline Head forward & $27.2 \pm 11.9 *$ & $15.6 \pm 9.7$ & $12.3 \pm 6.3^{*}$ & $3.7 \pm 2.6$ & $4.3 \pm 5.3^{*}$ & $1.3 \pm 1.0$ & $2.7 \pm 3.1^{*}$ & $0.8 \pm 0.2$ \\
\hline
\end{tabular}

Values are mean \pm standard deviation

Post-hoc Tukey test was applied among the different positions following a significance on the one-way ANOVA ( ${ }^{*} \mathrm{p}$ $<0.05)$ 
cally significant with $\mathrm{p}$-values $<0.05$.

\section{RESULTS}

Comparing the posturographic parameters at eight positions between the patient and control groups

The stability and weight distribution indices of the patients were significantly higher in all different positions (Table 1). The stability index while standing on pillows with eyes closed was more significantly increased than while standing with eyes open, or on pillows with eyes open. The weight distribution index was significantly higher when standing with eyes closed, or head backward than in standing on pillows.

The Fourier index of the patients was significantly increased at each different position at low, low-medium, and high frequencies. The Fourier index at a high frequency was significantly higher in standing on pillows with eyes closed, or with the head turning right/left/ backward/forward (Table 2). There was no significant difference of the synchronization indices between the patient and control groups in the eight positions (Table 3). The fall index of the patient group was $82.0 \pm 14.4$ (range 58-100), and significantly higher than that of the control group (8.2 \pm 4.2 , range 2-14).
Correlation between LEMS, K-BBS, TUG, K-MBI and posturographic parameters of the patient group

The average of LEMS was 32.5 \pm 5.2 (range 24-39), K-BBS $40.4 \pm 8.1$ (range 28-53), TUG $29.0 \pm 19.8$ seconds (range 8-62), and K-MBI 82.0 \pm 7.0 (range 72-93).

The fall index of patients did not significantly correlated with LEMS, but correlate with K-BBS, TUG, and K-MBI. The LEMS of the patients had a significant correlation with the synchronization indices between the toe and heel plates, and both toe plates (Table 4).

\section{DISCUSSION}

Posturographic parameters of the patients in quiet standing were compared with those of the healthy control group in this study. Higher stability and weight distribution indices of the patients could suggest a lack of overall stability and uneven weight distribution. Even when patients were standing with eyes open, postural sway was induced and transmitted to the foot-plates, causing poor stability and uneven weight distribution.

Significantly high stability, weight distribution, and falling indices regardless of positions suggested that patients had poor stability, uneven weight distribution, and a lack of overall balance. Even when patients were standing with eyes open and staring at the front wall, the stability was decreased and the weight distribution was asymmet-

Table 3. Comparison of Synchronization Indices in Eight Positions between the Patient and Control Group

\begin{tabular}{|c|c|c|c|c|c|c|c|c|}
\hline \multirow{2}{*}{ Position } & \multicolumn{2}{|c|}{ Left Toe-heel plates } & \multicolumn{2}{|c|}{ Right Toe-heel plates } & \multicolumn{2}{|c|}{ Toe-toe plates } & \multicolumn{2}{|c|}{ Heel-heel plates } \\
\hline & Patient & Control & Patient & Control & Patient & Control & Patient & Control \\
\hline Eyes open & $-728.9 \pm 229.5$ & $-824.5 \pm 204.0$ & $-767.6 \pm 417.3$ & $-874.2 \pm 102.5$ & $584.9 \pm 258.3$ & $694.7 \pm 203.0$ & $700.8 \pm 233.9$ & $845.1 \pm 120.1$ \\
\hline Eyes closed & $-800.6 \pm 158.7$ & $-903.6 \pm 92.5$ & $-690.5 \pm 354.0$ & $-894.4 \pm 75.5$ & $651.5 \pm 248.9$ & $732.7 \pm 221.7$ & $212.5 \pm 256.0$ & $860.6 \pm 95.4$ \\
\hline $\begin{array}{l}\text { Eyes open } \\
\text { on pillows }\end{array}$ & $-651.1 \pm 326.6$ & $-830.7 \pm 130.2$ & $-629.1 \pm 554.4$ & $-860.8 \pm 72.0$ & $554.3 \pm 479.3$ & $715.7 \pm 237.6$ & $607.3 \pm 339.2$ & $776.7 \pm 173.1$ \\
\hline $\begin{array}{l}\text { Eyes closed } \\
\text { on pillows }\end{array}$ & $-586.1 \pm 298.6^{*}$ & & $-549.6 \pm 562.9$ & $-894.8 \pm 67.0$ & $584.4 \pm 362.7$ & $820.0 \pm 70.4$ & $478.7 \pm 366.5^{*}$ & $856.0 \pm 90.0$ \\
\hline Head right & $-655.4 \pm 330.8$ & $-823.8 \pm 131.7$ & $-624.8 \pm 370.9$ & $-869.7 \pm 71.1$ & $492.5 \pm 292.4$ & $602.4 \pm 293.0$ & $587.5 \pm 369.2$ & $707.3 \pm 279.4$ \\
\hline Head left & $-690.2 \pm 243.4$ & $-802.9 \pm 145.8$ & $-695.3 \pm 374.1$ & $-793.1 \pm 156.5$ & $516.2 \pm 380.1$ & $629.8 \pm 205.5$ & $515.5 \pm 418.6$ & $723.1 \pm 262.6$ \\
\hline $\begin{array}{l}\text { Head } \\
\text { backward }\end{array}$ & $-790.0 \pm 121.4$ & $-848.9 \pm 75.8$ & $-716.6 \pm 317.9$ & $-879.1 \pm 91.4$ & $658.9 \pm 191.6$ & $663.5 \pm 150.1$ & $659.2 \pm 252.7$ & $801.9 \pm 108.4$ \\
\hline $\begin{array}{l}\text { Head } \\
\text { forward }\end{array}$ & $-726.6 \pm 230.2$ & $-857.4 \pm 120.8$ & $-772.2 \pm 258.7^{*}$ & *-895.5 \pm 80.4 & $433.5 \pm 496.3$ & $610.6 \pm 244.5$ & $656.5 \pm 377.3$ & $848.3 \pm 137.7$ \\
\hline
\end{tabular}

Values are mean \pm standard deviation

Post-hoc Tukey test was applied among the different positions following a significance on the one-way ANOVA $\left({ }^{*} \mathrm{p}<0.05\right)$ 
Table 4. Correlation between LEMS, K-BBS, TUG, K-MBI and Posturographic Parameters

\begin{tabular}{llllc}
\hline \multicolumn{2}{c}{ Posturographic parameter } & \multicolumn{1}{c}{ Position } & Correlation coefficient \\
\hline LEMS & Synchronization index & Left toe-heel & Eyes open & $0.67^{*}$ \\
& & Right toe-heel & Eyes open on pillows & $-0.70^{*}$ \\
& & & Head left & $-0.76^{*}$ \\
& & Heel-heel & Head forward & $-0.65^{*}$ \\
& & & Head left & $0.70^{*}$ \\
K-BBS & Fourier index & High frequency & Head backward & $0.66^{*}$ \\
TUG & Fall index & & & $0.81^{*}$ \\
& Synchronization index & Right toe-heel & Eyes open on pillows & $-0.72^{*}$ \\
& & Heel-heel & Head backward & $0.81^{*}$ \\
K-MBI & Fall index & & & $0.70^{*}$ \\
& Synchronization index & Toe-toe & Eyes open & $-0.60^{*}$ \\
& Fourier index & Low frequency & Head forward & $-0.71^{*}$ \\
\hline
\end{tabular}

LEMS: Lower extremity motor score, K-BBS: Korean version of Berg balance scale, TUG: Timed Up and Go Test, KMBI: Korean version of modified Barthel index

${ }^{*} \mathrm{p}<0.05$

ric because of postural sway. The stability of the patients was poorer in standing on pillows with eyes closed than those standing with eyes open, or standing on pillows with eyes open. The patients were more dependent on visual information to compensate for the somatosensory impairment when the unstable surface restricted the somatosensory sense. Therefore, a patients' increased visual restriction decreased their general stability.

In addition, an uneven weight distribution was more aggravated in standing with eyes closed, or with the head backward than in standing with the eyes open. In other words, instability worsened when visual compensation was insufficient, and their center of gravity moved posteriorly. It was considered that poor weight distribution was intensified with posterior migration of the center of gravity, which in turn could influence anteroposterior stability.

No significant difference of the synchronization index between the two groups might mean there was an adequate mirror-image compensation between the toe and heel plates, and parallel coactive synchronization between the bilateral toe plates, and heel plates.

A high Fourier index at low frequency could be caused by visual dysfunction. Since we excluded the patients with visual problems, a high index was likely because of improper visuo-vestibulo-equilibrium feedback. It seemed that the patients needed effortful control even in quiet standing. A high Fourier index at low-medium frequency may be more related to musculoskeletal fatigue than vestibular dysfunction, because patients with vestibular disorders were excluded from this study. Following SCI, there were reductions of type 1 and type $2 \mathrm{a}$ fibers; a loss of muscle bulk; a decrease in fiber-crosssectional areas; a reduction in torque; and increased fatigability. ${ }^{18}$ The mean LEMS of the patients were 32.5 \pm 5.2 . Because of the weakness of their lower-extremities, patients experienced more fatigability when maintaining a standing posture. Central nervous system dysfunction could make the Fourier index at a high-frequency higher. High Fourier index scores were represented by a gradual postural tremor. The spinal cord injury blockaded neural control and the transmission of posture and movement. The process of controlling posture and movement was initiated in the sensory association cortices of the parietal, temporal, and occipital lobes. These areas are projected to the frontal association cortex. The command to move was transmitted to the primary motor cortex and premotor cortex via the cerebellum and basal ganglia. The command for movement was transmitted to the spinal cord motor neurons, indirectly via the brain stem motor center, or directly via the corticospinal system. Motor signals from the spinal cord reached skeletal muscle via 
peripheral nerves and the neuromuscular junction. The motor system was controlled, coordinated, and integrated by neural regulating factors and neural networks not only from the corticospinal tract but also from the reticulospinal, vestibulospinal, and rubrospinal tracts, the cerebellum, and basal ganglia. ${ }^{19}$

In the Fourier index at a low-medium frequency, there was no significant difference between the two groups when the patients were standing with their eyes open, even while on pillows to restrict somatosensory function. However the index in standing with eyes closed was significantly higher than in the control group. Visual restriction could worsen the somatosensory impairments of patients. It was important to apply the visual input properly to compensate instability.

Lower-extremity power had significant correlation with several synchronization indices, and no correlation with stability, weight distribution, and fall indices. Two patients out of 10 scored grade 1 on the manual muscle test for the right ankle dorsiflexor, extensor hallucis longus, and ankle plantar flexor muscles. Other key muscles scored higher than grade 2 (mostly grades 3,4 ) on the manual muscle test.

As the LEMS increased for the right lower-extremity, the synchronization index between the right toe and heel plates declined. This meant that the lower-extremities power intensified the compensatory relationship between both plates. There was no correlation with the left side because left lower-extremity power showed little difference of manual muscle test grade scores within patients. Stronger muscle power was associated with higher synchronization indices between both toe plates, especially when standing with the head turned left, or backward. It seemed that increments of lower-extremity power helped synchronize reactions between those plates. Lower-extremity power and synchronization had significant correlation especially when the patients were standing without visual input, or with a posture to provoke instability. In circumstances to induce postural sway, the patients who had a more powerful strength of lower-extremities demonstrated better control of fine pedal movement.

LEMS had no significant correlation with stability, weight distribution, and fall indices. This suggests that muscular power alone was insufficient to maintain quiet standing because proprioceptive impairment was still an important factor affecting stability in quiet standing, evenness of weight distribution, and risk of falling.

Lower-extremity power was crucial to walk properly. It had significantly correlated with predictive parameters of ambulation. ${ }^{4,11}$ It has been one of the prime factors influencing postural balance. ${ }^{11}$ This study revealed that lower extremity power had a significant effect on postural balance of the patients, not in terms of overall stability and weight distribution, but, in terms of pedal synchronization.

K-BBS, TUG, and K-MBI showed a significant correlation with the fall index, and no correlation with other posturographic parameters. The fall index reflected the results of stability, Fourier, and synchronization indices. Such an overall balance index was affinitive to clinical balance tests, or activities of daily living. The fall index might be used to predict a possibility to ambulate, and to measure the independence of people with incomplete spinal cord injuries. Past studies have reported that postural instability could cause a dependency of daily activities, and a deterioration in the quality of life. ${ }^{2,11}$ The fall index was related to a possibility to move and walk safely, the degree to be assisted by caregivers, and the ability to live a quality life.

One of limitations of this study was that subjects of the patient group numbered only 10 people. We did a careful interpretation of datum to avoid a hasty generation mistake. Candidate recruiting was difficult because of the required inclusion criteria of this study. Patients with incomplete spinal cord injuries, some ability to walk, and with no specific conditions or diseases which could influence the study were few. We need to perform more studies and interpretations with a larger group of patients. Also, careful interpretations of the Fourier index was needed, because test-retest reliability was more insufficient than that of the stability index,,$^{20}$ even though it was theoretically possible.

\section{CONCLUSION}

The quiet standing balance of patients with incomplete cervical injuries was influenced by an unstable floor surface which could limit somatosensory input, and was further decreased by insufficient visual compensation. We should try to improve postural balance, gait, functional independence, and quality of life in incomplete spinal 
cord injury patients with proper proprioceptive and strength training for better postural and pedal control, making efforts to minimize environmental hazards.

\section{REFERENCES}

1. Patrick M, Ditunno PL, Dittuno JF. A comparison of spinal cord injury (SCI) consumers/staff preference for walking: a pilot study. J spinal Cord Med 2003; 26 : S41

2. Harkema SJ, Schmidt-Read M, Lorenz D, Edgerton VR, Behrman AL. Balance and ambulation improvements in individuals with chronic incomplete spinal cord injury using locomotor training-based rehabilitation. Arch Phys Med Rehabil 2011; 20 Suppl 1: S1-10

3. Kim MO, Jung HY, Lee JJ, Lee JH, Jeong HJ, Joa KL. The significance of the Berg balance scale as a parameter of walking outcome in post-acute spinal cord injured patients. J Korean Acad Rehab Med 2010; 34: 513-517

4. Morganti B, Scivoletto G, Ditunno P, Ditunno J, Molinari M. Walking index for spinal cord injury (WISCI): criterion validation. Spinal Cord 2004; 43: 27-33

5. Jacobson GP, Newman CW, Kartush JM. Handbook of balance function testing, San Diego: Singular Pub Group, 1993, 259-307

6. Nashner LM, Peters JF. Dynamic posturography in the diagnosis and management of dizziness and balance disorders. Neurol Clin 1990; 8: 331-349

7. Yelnik A, Bonan I. Clinical tools for assessing balance disorders. Neurophysiol Clin 2008; 38: 439-445

8. Kim CR, Chun MH, Lee GA. Assessments of balance control using tetra-ataxiametric posturography. J Korean Acad Rehab Med 2009; 33: 429-435

9. Kim BR, Choi KH, Chun MH, Lee MC, Chung SJ, Jang $\mathrm{KW}$. Evaluation of balance control in patients with idiopathic Parkinson's disease using tetra-ataxiometric posturography. J Korean Acad Rehab Med 2009; 33: 538-546

10. Blum L, Korner-Bitensky N. Usefulness of the Berg
Balance Scale in stroke rehabilitation: a systematic review. Phys Ther 2008; 88: 559-566

11. Ditunno JF Jr, Barbeau H, Dobkin BH, Elashoff R, Harkema S, Marino RJ, Hauck WW, Apple D, Basso DM, Behrman A, et al. Validity of the walking scale for spinal cord injury and other domains of function in a multicenter clinical trial. Neurorehabil Neural Repair 2007; 21: 539-550

12. Podsiadlo D, Richardson S. The timed" Up \& Go": a test of basic functional mobility for frail elderly persons. J Am Geriatr Soc 1991; 39: 142-148

13. Van Hedel HJ, Wirz M, Dietz V. Assessing walking ability in subjects with spinal cord injury: validity and reliability of 3 walking tests. Arch Phys Med Rehabil 2005; 86: 190-196

14. Wirz M, Zemon DH, Rupp R, Scheel A, Colombo G, Dietz V, Hornby TG. Effectiveness of automated locomotor training in patients with chronic incomplete spinal cord injury: a multicenter trial. Arch Phys Med Rehabil 2005; 86: 672-680

15. Hocking C, Williams M, Broad J, Baskett J. Sensitivity of Shah, Vanclay and Cooper's modified Barthel Index. Clin Rehabil 1999; 13: 141-147

16. Jung HY, Park BK, Shin HS, Kang YK, Pyun SB, Paik NJ, Kim SH, Kim TH, Han TR. Development of the Korean version of modified Barthel index (K-MBI): Multicenter study for subjects with stroke. J Korean Acad Rehab Med 2007; 31: 283-297

17. Chon JM, Kim HS, Yoo SD, Kim DH, Shin YW, Yun DH. Assessment of fall risk in patients with osteoporosis by posturography. Clinical pain 2010; 9: 27-31

18. Braddom RL. Physical medicine and rehabilitation, 3rd ed, Philadelphia: Elsevier Inc., 2007, 489

19. Afifi AK. Bergman RA. Functional neuroanatomy, 2nd ed, New York: Mc Graw Hill, 2005, 412-419

20. Kohen-Raz R. Application of tetra-ataxiametric posturography in clinical and developmental diagnosis. Percept Mot Skills 1991; 73: 635-656 